\title{
The effect of gonadal and extragonadal malignant cancers on sperm quality
}

\author{
Xiao Liu ${ }^{1}$, Bo Liu ${ }^{1}$, Shasha Liu ${ }^{1}$, Yang Xian ${ }^{1}$, Wenrui Zhao ${ }^{1}$, Min Jiang ${ }^{1}$, Bin Zhou ${ }^{2}$, Fuping Li $^{1}$ \\ ${ }^{1}$ Human Sperm Bank, Key Laboratory of Birth Defects and Related Diseases of Women and Children (Sichuan University), Ministry of \\ Education, West China Second University Hospital, Sichuan University, Chengdu, China; ${ }^{2}$ Laboratory of Molecular Translational Medicine, \\ Center for Translational Medicine, Key Laboratory of Birth Defects and Related Diseases of Women and Children (Sichuan University), Ministry \\ of Education, Clinical Research Center for Birth Defects of Sichuan Province, West China Second University Hospital, Sichuan University, \\ Chengdu, China \\ Contributions: (I) Conception and design: X Liu, F Li; (II) Administrative support: B Zhou; (III) Provision of study materials or patients: B Liu; (IV) \\ Collection and assembly of data: S Liu, Y Xian; (V) Data analysis and interpretation: W Zhao, M Jiang; (VI) Manuscript writing: All authors; (VII) \\ Final approval of manuscript: All authors. \\ Correspondence to: Fuping Li, PhD. Human Sperm Bank, West China Second University Hospital, Sichuan University, No. 1416, Section 1, \\ Chenglong Avenue, Chengdu 610066, China. Email: Ifpsnake@scu.edu.cn.
}

\begin{abstract}
Background: Many studies have reported that testicular germ cell tumours (TGCTs) give rise to a decrease in sperm production and may further lead to infertility. However, little is known regarding sperm production in extragonadal germ cell tumours (EGCTs). We have studied the differences in the characteristics and sperm quality of patients with testicular cancer and patients with EGCTs.

Methods: We collected and analysed demographic factors, semen parameters, and the disposition of banked sperm.

Results: TGCTs accounted for the majority of malignant cancers compared with EGCTs $(83.9 \%$ vs. $16.1 \%$, respectively). There were no statistically significant differences in semen volume, pre-freeze concentration, pre-freeze motility, post-thaw concentration, post-thaw motility or recovery rate between patients with TGCTs and those with EGCTs. Both patients with TGCTs and EGCTs showed low prefreeze and post-thaw sperm concentrations. Men with a seminoma had a higher median level of thaw-sperm motility in their ejaculate than men with non-seminomas. TGCT (67\%) and EGCT (72\%) patients chose to keep their specimens in storage.

Conclusions: Our study provides evidence that both patients with TGCTs and EGCTs have a high risk for impaired semen quality and gonadal dysfunction. This suggests that oncologists and patients should consider the effects of both TGCTs and EGCTs on fertility among males.
\end{abstract}

Keywords: Testicular germ cell tumours (TGCTs); extragonadal germ cell tumours (EGCTs); semen analysis; cryopreservation; spermatozoa

Submitted Aug 20, 2021. Accepted for publication Nov 10, 2021.

doi: $10.21037 /$ tcr-21-1697

View this article at: https://dx.doi.org/10.21037/tcr-21-1697

\section{Introduction}

Human germ cell tumours (GCTs) originate from stem cells of the germ line or the early embryo. GCTs are classified into testicular germ cell tumours (TGCTs) or extragonadal germ cell tumours (EGCTs), as they occur in the gonads (ovaries and testes) or in extragonadal sites.

EGCTs are a heterogeneous group of neoplasms, and can arise virtually anywhere, but typically the site of origin is in the midline of the body, including the sacral, retroperitoneal and mediastinal regions and the midline of the brain (1). The aetiology of a malignant tumour is considered multifactorial, 
including abnormalities in early embryonic development and chromosomal abnormalities (1). There is little research on EGCTs and their effects of human reproductive health. Only a few studies have examined these tumours in the form of case reports or case series (2). Patients with EGCTs showed a significant lower sperm concentration and lower inhibin $\mathrm{B}$ levels than those patients with other malignancies as seen in the Haematological malignancy and Sarcoma's groups (3). Jacobsen et al. showed that low semen quality is a risk factor for patients with EGCTs (4).

Testicular cancer, which accounts for only $1 \%$ of all cancers, is one of the most common malignancies in young adult men, with a maximum age range of $20-44$ years $(5,6)$, and the overall survival rate is up to more than $95 \%$ of patients being cured (7-9). Because of the high survival rates, the preservation of fertility potential is a particularly important consideration for the patients before the treatment as testicular tumours might affect spermatogenesis, and the semen quality of patients is generally decreased $(3,10,11)$. Testicular cancer patients are more likely to see a fertility specialist than men with other cancers, possibly because they had already been treated by a urologist (12). Guidelines published by the American Society of Clinical Oncology (ASCO) recommend that the discussion about fertility preservation with patients should be initiated as soon as possible after the initial diagnosis of cancer (13). Treatment options for malignant GCTs include surgery and chemotherapy. Some studies have reported that sperm count and motility are significantly lower in men with testicular tumours. One study reported that $50.0 \%$ of patients presented with a decrease in sperm concentration after orchiectomy. Even before surgery, approximately half of patients with TGCTs presented poor semen quality (14).

Compared to EGCTs, there are many studies on TGCTs and semen quality. However, there is little material about the differences in sperm parameters between patients with EGCTs and those with TGCTs. To determine the differences between them, we analysed demographic factors, semen parameters and the disposition of banked sperm in the Human Sperm Bank, West China Second University Hospital, Sichuan University between 2010 and 2019. The aim of this study was to describe the effects of TGCTs and EGCTs on spermatogenesis and semen quality. It is important to pay attention to the fertility conservation of GCTs, especially among young men. We present the following article in accordance with the STROBE reporting checklist (available at https://dx.doi.org/10.21037/tcr-211697).

\section{Methods}

\section{Patient population}

In accordance with the guidelines and processes established by our sperm bank, 87 cancer patients seeking fertility preservation were counselled by an andrology physician and fully informed about the procedure. The information provided included the different methods of fertility preservation and the procedures for the future use of the specimens. All patients received a physical examination. The medical records of all cancer patients who attempted sperm banking at our Human Sperm Bank from January 2010 to December 2019 were reviewed. These data included the following: age at diagnosis, cancer type, preand post-cryopreservation semen analysis results, the number of straws stored, current banking status, usage of the frozen semen, and the reproductive outcome of using frozen/thawed spermatozoa. The disposition categories were ongoing storage within our unit, electively discarded, failure to bank, used, and death. Many types of cancer were determined by the oncologist's letter or histological diagnosis. However, there are still some cancer diagnoses unavailable, and we followed up those cancer patients by our phone call and some patients can't get through or don't answer our phone. Moreover, some patients come to our unit to freeze sperm by themself rather than being recommended by a doctor. So, some patients' definite diagnoses are unavailable. The study was conducted in accordance with the Declaration of Helsinki (as revised in 2013). This study was approved by the Ethics Committee of the West China Second University Hospital (No. 2020001), and individual consent for this retrospective analysis was waived.

\section{Semen samples}

Semen samples were obtained by masturbation into sterile containers. All the samples were placed in an incubator at $37{ }^{\circ} \mathrm{C}$ until liquefaction and were subsequently analysed within 1 hour. The following semen parameters were evaluated according to the World Health Organization (WHO) 1999, 2010 guidelines: sperm concentration, volume and progressive motility (grades a $+\mathrm{b})(15,16)$.

Cryopreservation of the spermatozoa was performed as follows. Sperm cryopreservation was performed as follows. The liquefied semen sample was transferred to a $2.0 \mathrm{~mL}$ sterile cryotube with Sperm Cryoprotectant Kit [Anhui Anke Biotechnology (Group) Co., Ltd., China], glycerol- 


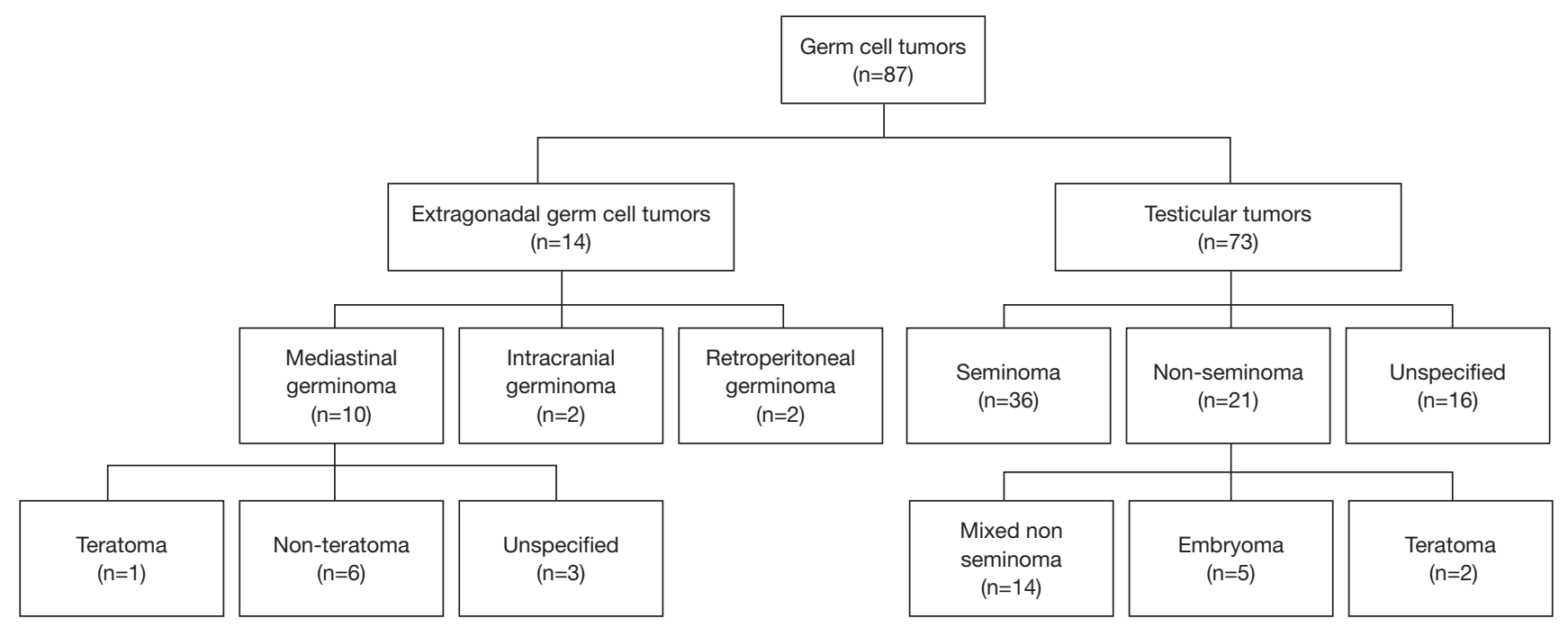

Figure 1 Flow diagram of the GCT patients who presented to Sichuan human sperm bank from 2010 to 2019 for sperm cryopreservation. GCT, germ cell tumour.

egg yolk free cryopreservative medium, final glycerol concentration of $7.5 \%(17,18)$ added dropwise until a 3:1 sample: medium ratio. The final volume of diluted seminal fluid in a tube was $1 \mathrm{~mL}$. Then straws were stored at $4{ }^{\circ} \mathrm{C}$ for 15 minutes and then suspended above of liquid nitrogen $5 \mathrm{~cm}$ for 10 minutes before being stored in liquid nitrogen. For semen thawing, straws were placed at room temperature for 1 minute and then were transferred to a beaker containing $37{ }^{\circ} \mathrm{C}$ water until all ice crystals had disappeared. A diagnosis of azoospermia was confirmed by centrifugation at $3,000 \mathrm{~g}$ for 15 minutes of the entire semen sample.

\section{Statistical analysis}

The statistical methodology consisted of Mann-Whitney test and Student's $t$-test. In all cases, $\mathrm{P}<0.05$ was considered statistically significant.

\section{Results}

\section{Population studied}

There were 87 males with GCTs who attempted sperm cryopreservation from January 2010 to December 2019. GCTs included 14 EGCTs (16.1\%) and 73 TGCTs (83.9\%) (Figure 1). EGCTs were classified into mediastinal germinoma (10/14), intracranial germinoma (2/14) and retroperitoneal germinoma (2/14). Mediastinal germinoma was the most common type of EGCT in our unit, which included 1 teratoma, 6 non-teratoma and 3 unspecified. There are two subtypes of TGCT, namely, seminoma and non-seminoma $(19,20)$. Seminomas are the most usually diagnosed type of testicular cancer, accounting for approximately half of all cases $(19,21)$. Non-seminomas include elements of teratoma, embryonal carcinoma, yolk sac tumour and choriocarcinoma. We collected 36 seminomas and 21 non-seminomas to conduct sperm cryopreservation. Moreover, there were 16 TGCTs that could not be definitively diagnosed as seminomas or nonseminomas due to failing to follow up cancer patients by telephone, so they were labelled as unspecified. Non-seminomas included 14 mixed non-seminomas, 5 embryonal carcinomas, and 2 teratomas in our institution (Figure 1).

\section{Semen parameters}

Median sexual abstinence was in the range of at least 2 days and at most 7 days (22). However, a few of the patients had a longer period of sexual abstinence than 7 days due to emergency treatment (data not shown). The mean age at banking was statistically significant difference in patients with TGCTs and those with EGCTs. However, there was no statistical significance in semen volume, pre-freeze concentration, pre-freeze motility, post-thaw concentration, post-thaw motility or recovery rate between the two types. 
Table 1 Demographics and semen parameters of patients with GCT

\begin{tabular}{|c|c|c|c|c|}
\hline Parameters & TGCTs & EGCTs & Lower reference limits ${ }^{\dagger}$ & $P$ value ${ }^{c}$ \\
\hline Volume at banking $(\mathrm{mL})^{\mathrm{a}}$ & $3.8 \pm 1.6$ & $3.4 \pm 1.6$ & $1.5[1.4-1.7]$ & $0.5303^{d}$ \\
\hline Pre-count $\left(10^{6} / \mathrm{mL}\right)^{\mathrm{b}}$ & $25.5[0.9-159]$ & $26[2-105]$ & $15[12-16]$ & $0.6264^{\mathrm{e}}$ \\
\hline Pre-progressive motility (\%) & 49 [3.1-83] & $48[13-70]$ & $40[38-42]$ & $0.5206^{\mathrm{e}}$ \\
\hline Post-motility (\%) & 28 [2.6-61] & $31[0.66-58]$ & NA & $0.9277^{e}$ \\
\hline Recovery rates $(\%)^{\mathrm{b}}$ & $59.5[6.8-89.6]$ & $58.4[2.2-84.6]$ & NA & $0.9136^{e}$ \\
\hline
\end{tabular}

${ }^{a}$, mean \pm standard deviation; ${ }^{b}$, median [range]; ${ }^{c}, \mathrm{P}<0.05$ significant; ${ }^{\mathrm{d}}, t$-test was used; ${ }^{\mathrm{e}}$, Mann-Whitney test was used; ${ }^{\mathrm{f}}$, lower reference limits: 5th centiles and their 95\% confidence intervals according to the WHO 2010 guidelines. Pre-concentration and pre-progressive motility: count of spermatozoa (million/mL) and percentage of spermatozoa with progressive motility before banking; post-count and post-progressive motility: count of spermatozoa (million $/ \mathrm{mL}$ ) and percentage of spermatozoa with progressive motility after thawing; recovery rate (\%): the percentage recovery of progressively motile spermatozoa after thawing as compared to fresh spermatozoa before banking. GCT, germ cell tumour; TGCTs, testicular germ cell tumours; EGCTs, extragonadal germ cell tumours; WHO, World Health Organization.

Table 2 Characteristics of patients with seminomatous and non-seminomatous TGCTs

\begin{tabular}{|c|c|c|c|c|}
\hline Parameters & Seminoma & Non-seminoma & Lower reference limits $^{\dagger}$ & $P$ value ${ }^{c}$ \\
\hline Volume at banking $(\mathrm{mL})^{\mathrm{a}}$ & $3.7 \pm 1.6$ & $3.7 \pm 1.6$ & $1.5[1.4-1.7]$ & $0.9727^{d}$ \\
\hline Pre-count $\left(10^{6} / \mathrm{mL}\right)^{b}$ & $19.5[1.5-64]$ & 33 [3.4-159] & $15[12-16]$ & $0.1614^{\mathrm{e}}$ \\
\hline Pre-motility $(\%)^{\mathrm{b}}$ & 54.5 [9-83] & $50[10-74]$ & $40[38-42]$ & $0.2414^{\mathrm{e}}$ \\
\hline Post-motility $(\%)^{b}$ & $36.5[6-51]$ & 24.5 [3-61] & NA & $0.0187^{\mathrm{e}}$ \\
\hline Recovery rates $(\%)^{\mathrm{b}}$ & $61.9[17.1-77.8]$ & 56.7 [28.6-89.60] & NA & $0.2761^{\mathrm{e}}$ \\
\hline
\end{tabular}

${ }^{\mathrm{a}}$, mean \pm standard deviation; ${ }^{\mathrm{b}}$, median [range]; ${ }^{\mathrm{c}}, \mathrm{P}<0.05$ significant; ${ }^{\mathrm{d}}, t$-test was used; ${ }^{\mathrm{e}}$, Mann-Whitney test was used; ${ }^{\mathrm{f}}$, lower reference limits: 5 th centiles and their $95 \%$ confidence intervals according to the WHO 2010 guidelines. Pre-concentration and pre-progressive motility: count of spermatozoa (million/mL) and percentage of spermatozoa with progressive motility before banking; post-count and post-progressive motility: count of spermatozoa $(\mathrm{million} / \mathrm{mL}$ ) and percentage of spermatozoa with progressive motility after thawing; recovery rate (\%): the percentage recovery of progressively motile spermatozoa after thawing as compared to fresh spermatozoa before banking. TGCTs, testicular germ cell tumours; WHO, World Health Organization.

Both patients with TGCTs and EGCTs showed low pre-freeze and post-thaw sperm concentrations. Semen characteristic evaluation per diagnosis is listed in Table 1. The median sperm concentration was $19.5 \times 10^{6} / \mathrm{mL}$ in the seminoma group and $33 \times 10^{6} \% \mathrm{~mL}$ in the nonseminoma group; this difference was not significant. Prefreeze motility was also not significantly different between the seminoma group $(54.5 \%)$ and the non-seminoma group $(50 \%)$ irrespective of tumour stage (Table 2). However, the non-seminoma group (24.5\%) had significantly lower median levels of post-thaw sperm motility than the seminoma group (36.5\%). Statistical analyses did not reveal significant differences in sperm count or recovery rates between those two groups (Table 2).

\section{Disposition of cryopreserved sperm}

The fate of cryopreserved sperm in testicular cancer categories included ongoing storage (67\%), electively discarded (24\%), failure to bank (7\%), and used (2\%) 

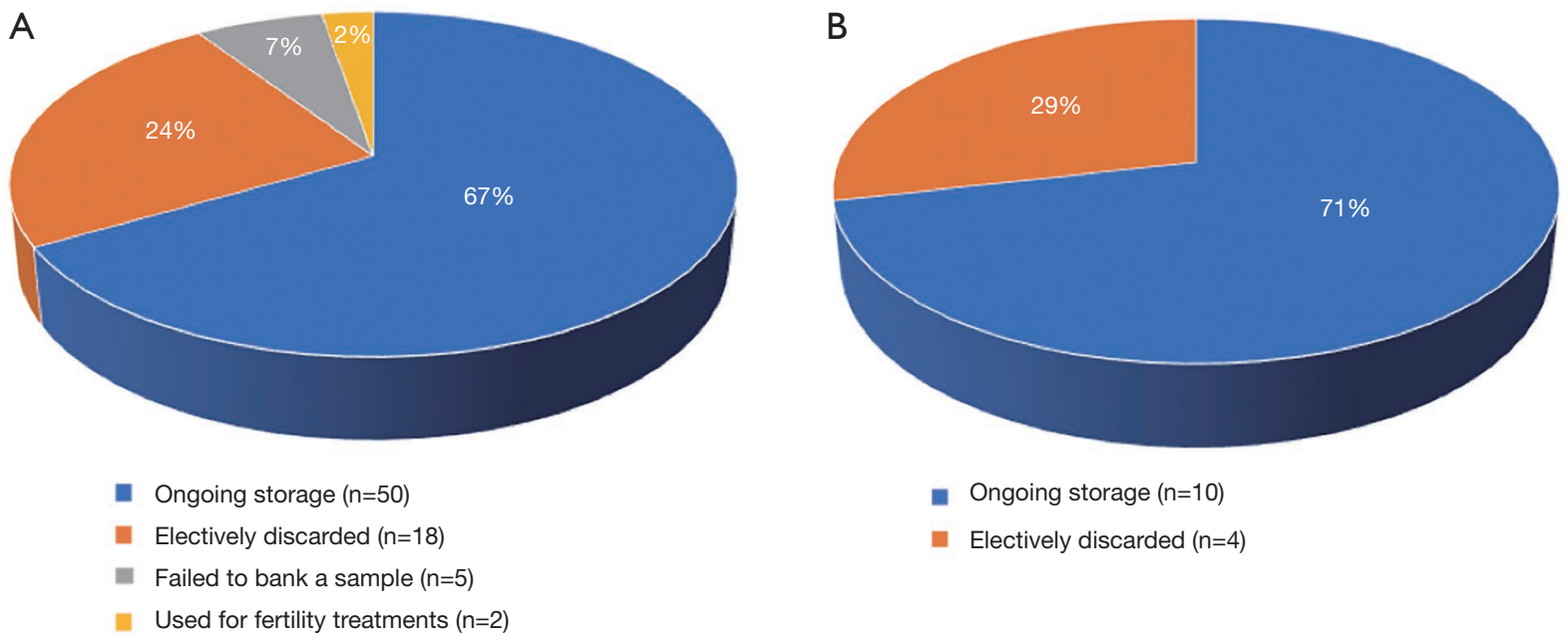

Figure 2 Outcomes for the 87 GCT patients who attempted banking. (A) Outcomes of patients with TGCTs who attempted banking. (B) Outcomes of patients with EGCTs who attempted banking. GCT, germ cell tumour; TGCTs, testicular germ cell tumours; EGCTs, extragonadal germ cell tumours.

(Figure 2A). Five TGCT patients exhibited azoospermia (three seminomas, one mixed non-seminoma, one embryoma) and failed to perform sperm cryopreservation.

Onco-testicular sperm extraction (onco-TESE) could not be provided at the time of orchiectomy because of a shortage of embryologists and urologists to perform the emergency TESE. Conceptions were achieved in $61.5 \%$ (8 out of 13), with $38.5 \%$ (5 out of 13 ) of the pregnancies resulting in delivery. Two testicular cancers used cryopreserved semen for ART, and both couples had their babies.

The fate of cryopreserved sperm of EGCTs only included ongoing storage (71\%) and electively discarded (29\%) (Figure 2B). However, no EGCT patients applied to have their semen used for ART.

\section{Discussion}

Tumours in men could be classified as two groups based on their location: TGCT and EGCT. Testicular cancer includes both seminoma and non-seminoma. Seminomas are the most commonly diagnosed type of testicular cancer, accounting for approximately half of all cases. Nonseminoma can be pure or histologically mixed to include elements of embryonal carcinoma, teratoma, yolk sac tumour, and choriocarcinoma (21). EGCTs are histologically heterogeneous and are more common among male children aged 15 to 19 years $(23,24)$. In our study, we found that the
EGCT group were younger than the TGCT group. So, this may be one of the reasons accounting for the fact that the EGCT group were significantly younger. EGCTs are commonly hypothesized to arise from abnormal primordial germ cell migration during embryogenesis (25) and are often seen in the sacral, retroperitoneal and mediastinal regions and the midline of the brain (1). Moreover, EGCTs are uncommon tumours, and most descriptions of these tumours are in the form of case reports.

Many studies have examined TGCTs. Surgical treatment of testicular cancer such as orchiectomy has an even greater effect on fertility (26). Two studies showed that no differences in semen analysis were observed between pre- and post-orchiectomy TGCT groups $(10,14)$. So, although patients with testicular cancer are generally recommended to initiate therapy as soon as possible, there is still plenty of time in which semen cryopreservation may be performed. Our data are consistent with the literature, in that a significant reduction in sperm motility and concentration were observed in patients with testicular cancer $(11,27,28)$. Our findings were that the post-thaw motility is significantly lower in the non-seminoma group than seminoma group. However, it is not yet known if testicular cancer histology may determine different alterations to semen quality. Although some investigators show that non-seminoma patients showed a greater decrease than seminoma patients the total number of sperm with progressive motility, other investigators did 
not find that difference $(10,29,30)$. However, one study suggested that men with a non-seminoma presented with a lower number of motile morphologically normal sperm in the ejaculate than men with a seminoma (10). So, if sperm from non-seminoma are frozen and then thawed, the samples may show lower post-thaw motility than do sperm from seminoma patients.

In our study, both patients with TGCTs and EGCTs showed low pre-freeze and post-thaw sperm concentrations, and the endocrinology may the one reason for this. We tried to follow up with the referring clinic, but the patients were treated in different hospitals and the time interval is long, so it is difficult for us to follow up with the referring clinic about endocrinology. According to the literature, van Casteren et al. showed inhibin B levels were found significantly lower in the patients diagnosed with EGCTs than in those with TGCTs (3). In addition, the production of human chorionic gonadotropin (hCG) by the tumour may inhibit spermatogenesis (31). We plan to check the hormone levels, such as follicle-stimulating hormone (FSH), luteinizing hormone (LH), testosterone (T), estradiol (E2), inhibin B and hCG in our unit in future to study the root cause of low concentration.

Although the survival rates of GCTs, especially TGCTs, have increased, this low utilization rate is caused by various psychological and sociological factors (6). A recent systematic review of 30 studies showed that the utilization rate of preserved sperm is only $10 \%$ in those who bank sperm (32). However, over the course of 10 years, $2 \%$ of the patients who had TGCTs used their cryopreserved semen from our facility, and no EGCT patients returned to use in EGCTs. The fact that GCT patients were relatively young and had no plan to have babies might be the main reason for the low usage rate. Cost may be another barrier to the rate of sperm utilization. For patients with EGCTs, disease progression and poor prognosis accounted for the lack of use. Therefore, for patients with cancers, the usage rate of banked sperm is an issue that might be as important as the sperm quality. Further research is necessary to answer questions about how to manage the fertility of survivors of cancers.

\section{Conclusions}

In conclusion, based on our existing research, it may be speculated that semen samples from patients with TGCTs and EGCTs have a high risk of gonadal dysfunction and impaired semen quality. This suggests that we should pay more attention to the fertility conservation of young men with GCTs.

\section{Acknowledgments}

We would like to thank Jun Zhang for his help in polishing our paper.

Funding: This study was supported by Sichuan Science and Technology Program (No. 2020ZYD007).

\section{Footnote}

Reporting Checklist: The authors have completed the STROBE reporting checklist. Available at https://dx.doi. org/10.21037/tcr-21-1697

Data Sharing Statement: Available at https://dx.doi. org/10.21037/tcr-21-1697

Conflicts of Interest: All authors have completed the ICMJE uniform disclosure form (available at https://dx.doi. org/10.21037/tcr-21-1697). The authors have no conflicts of interest to declare.

Ethical Statement: The authors are accountable for all aspects of the work in ensuring that questions related to the accuracy or integrity of any part of the work are appropriately investigated and resolved. The study was conducted in accordance with the Declaration of Helsinki (as revised in 2013). This study was approved by the Ethics Committee of the West China Second University Hospital (No. 2020-001), and individual consent for this retrospective analysis was waived.

Open Access Statement: This is an Open Access article distributed in accordance with the Creative Commons Attribution-NonCommercial-NoDerivs 4.0 International License (CC BY-NC-ND 4.0), which permits the noncommercial replication and distribution of the article with the strict proviso that no changes or edits are made and the original work is properly cited (including links to both the formal publication through the relevant DOI and the license). See: https://creativecommons.org/licenses/by-nc-nd/4.0/.

\section{References}

1. Oosterhuis JW, Looijenga LHJ. Human germ cell tumours from a developmental perspective. Nat Rev Cancer 
2019;19:522-37.

2. Sun H, Ding H, Wang J, et al. The differences between gonadal and extra-gonadal malignant teratomas in both genders and the effects of chemotherapy. BMC Cancer 2019;19:408.

3. van Casteren NJ, Boellaard WP, Romijn JC, et al. Gonadal dysfunction in male cancer patients before cytotoxic treatment. Int J Androl 2010;33:73-9.

4. Jacobsen R, Bostofte E, Engholm G, et al. Risk of testicular cancer in men with abnormal semen characteristics: cohort study. BMJ 2000;321:789-92.

5. Moody JA, Ahmed K, Yap T, et al. Fertility managment in testicular cancer: the need to establish a standardized and evidence-based patient-centric pathway. BJU Int 2019;123:160-72.

6. Hamano I, Hatakeyama S, Ohyama C. Fertility preservation of patients with testicular cancer. Reprod Med Biol 2017;16:240-51.

7. Dearnaley D, Huddart R, Horwich A. Regular review: Managing testicular cancer. BMJ 2001;322:1583-8.

8. Brydøy M, Fosså SD, Klepp O, et al. Paternity following treatment for testicular cancer. J Natl Cancer Inst 2005;97:1580-8.

9. Haugnes HS, Bosl GJ, Boer H, et al. Long-term and late effects of germ cell testicular cancer treatment and implications for follow-up. J Clin Oncol 2012;30:3752-63.

10. Fraietta R, Spaine DM, Bertolla RP, et al. Individual and seminal characteristics of patients with testicular germ cell tumors. Fertil Steril 2010;94:2107-12.

11. Auger J, Sermondade N, Eustache F. Semen quality of 4480 young cancer and systemic disease patients: baseline data and clinical considerations. Basic Clin Androl 2016;26:3.

12. Hotaling JM, Lopushnyan NA, Davenport M, et al. Raw and test-thaw semen parameters after cryopreservation among men with newly diagnosed cancer. Fertil Steril 2013;99:464-9.

13. Oktay K, Harvey BE, Partridge AH, et al. Fertility Preservation in Patients With Cancer: ASCO Clinical Practice Guideline Update. J Clin Oncol 2018;36:19942001.

14. Andrade MBR, Bertolla RP, Intasqui P, et al. Effect of orchiectomy on sperm functional aspects and semen oxidative stress in men with testicular tumours. Andrologia 2019;51:e13205.

15. World Health Organization. WHO Laboratory Manual for the Examination of Human Semen and Sperm-Cervical Mucus Interaction. 4th ed. Cambridge: Cambridge
University Press, 1999.

16. World Health Organization. WHO Laboratory Manual for the Examination and Processing of Human Semen. 5th ed. Geneva: WHO Press, 2010.

17. Hammadeh ME, Greiner S, Rosenbaum P, et al. Comparison between human sperm preservation medium and TEST-yolk buffer on protecting chromatin and morphology integrity of human spermatozoa in fertile and subfertile men after freeze-thawing procedure. J Androl 2001;22:1012-8.

18. Mahadevan M, Trounson AO. Effect of cryoprotective media and dilution methods on the preservation of human spermatozoa. Andrologia 1983;15:355-66.

19. Sineath RC, Mehta A. Preservation of Fertility in Testis Cancer Management. Urol Clin North Am 2019;46:341-51.

20. Weibring K, Nord C, Ståhl O, et al. Sperm count in Swedish clinical stage I testicular cancer patients following adjuvant treatment. Ann Oncol 2019;30:604-11.

21. Cheng L, Albers P, Berney DM, et al. Testicular cancer. Nat Rev Dis Primers 2018;4:29.

22. Lu JC, Huang YF, Lü NQ. WHO Laboratory Manual for the Examination and Processing of Human Semen: its applicability to andrology laboratories in China. Zhonghua Nan Ke Xue 2010;16:867-71.

23. Poynter JN, Amatruda JF, Ross JA. Trends in incidence and survival of pediatric and adolescent patients with germ cell tumors in the United States, 1975 to 2006. Cancer 2010;116:4882-91.

24. Williams LA, Pankratz N, Lane J, et al. Klinefelter syndrome in males with germ cell tumors: A report from the Children's Oncology Group. Cancer 2018;124:3900-8.

25. Schneider DT, Schuster AE, Fritsch MK, et al. Multipoint imprinting analysis indicates a common precursor cell for gonadal and nongonadal pediatric germ cell tumors. Cancer Res 2001;61:7268-76.

26. Albers $\mathrm{P}$, Albrecht W, Algaba F, et al. Guidelines on Testicular Cancer: 2015 Update. Eur Urol 2015;68:1054-68.

27. Johnson MD, Cooper AR, Jungheim ES, et al. Sperm banking for fertility preservation: a 20-year experience. Eur J Obstet Gynecol Reprod Biol 2013;170:177-82.

28. Meseguer M, Molina N, García-Velasco JA, et al. Sperm cryopreservation in oncological patients: a 14-year followup study. Fertil Steril 2006;85:640-5.

29. Lass A, Akagbosu F, Abusheikha N, et al. A programme of semen cryopreservation for patients with malignant disease in a tertiary infertility centre: lessons from 8 years' experience. Hum Reprod 1998;13:3256-61. 
30. Hallak J, Kolettis PN, Sekhon VS, et al. Sperm cryopreservation in patients with testicular cancer. Urology 1999;54:894-9.

31. Kuczyk M, Machtens S, Bokemeyer C, et al. Sexual function and fertility after treatment of testicular cancer.
Curr Opin Urol 2000;10:473-7.

32. Ferrari S, Paffoni A, Filippi F, et al. Sperm cryopreservation and reproductive outcome in male cancer patients: a systematic review. Reprod Biomed Online 2016;33:29-38.

Cite this article as: Liu X, Liu B, Liu S, Xian Y, Zhao W, Jiang M, Zhou B, Li F. The effect of gonadal and extragonadal malignant cancers on sperm quality. Transl Cancer Res 2021;10(12):5183-5190. doi: 10.21037/tcr-21-1697 